\title{
Dextroplantation of a reduced left lateral section graft in an infant undergoing living donor liver transplantation
}

\author{
Shin Hwang, Jung-Man Namgoong, Gil-Chun Park, Kyung Mo Kim, Seak Hee Oh
}

Department of Surgery, Asan Medical Center, University of Ulsan College of Medicine, Seoul, Korea

Background: Graft size matching is essential for successful liver transplantation in infant recipients.

Methods: We present our technique of graft dextroplantation used in an infant who underwent living donor liver transplantation (LDLT) using a reduced left lateral section (LLS) graft.

Results: The patient was an 11-month-old female infant weighing $7.8 \mathrm{~kg}$ with hepatoblastoma. She was partially responsive to systemic chemotherapy. Thus, LDLT was performed to treat the tumor. The living donor was a 34 -year-old mother of the patient. After non-anatomical size reduction, the weight of the reduced LLS graft was $235 \mathrm{~g}$, with a graft-to-recipient weight ratio of $3.0 \%$. Recipient hepatectomy was performed according to the standard procedures of pediatric LDLT. At the beginning of graft implantation, the graft was temporarily placed at the abdomen to determine the implantation location. The graft portal vein was anastomosed with an interposed external iliac vein homograft. As the liver graft was not too large and it was partially accommodated in the right subphrenic fossa, thus the abdominal wall wound was primarily closed. The patient recovered uneventfully. An imaging study revealed deep accommodation of the graft within the right subphrenic fossa. The patient has been doing well for 6 months without any vascular complications.

Conclusions: This case suggests that dextroplantation of a reduced LLS graft can be a useful technical option for LDLT in infant patients.

Corresponding author: Shin Hwang

E-mail: shwang@amc.seoul.kr

(c) The Korean Society for Transplantation

This is an Open Access article distributed under the terms of the Creative Commons Attribution Non-Commercial License (http://creativecommons.org/licenses/by-nc/4.0/) which permits unrestricted non-commercial use, distribution, and reproduction in any medium, provided the original work is properly cited. 